\title{
SOME WHITEHEAD PRODUCTS ON ODD SPHERES
}

\section{MAURICE GILMORE}

1. Introduction. We consider the Whitehead product $\left[\iota_{n}, \iota_{n}\right]$ $\in \Pi_{2 n-1}\left(S^{n}\right)$ where $\iota_{n}$ is a generator of $\Pi_{n}\left(S^{n}\right)$. For $n$ even, it is well known that $\left[\iota_{n}, \iota_{n}\right]$ generates an infinite cyclic subgroup of $\Pi_{2 n-1}\left(S^{n}\right)$. Furthermore, this subgroup is actually a direct summand unless $n=2,4$ or 8 . For $n$ odd, $\left[\iota_{n}, \iota_{n}\right]$ generates a subgroup of order two. In [3], G. V. Krishnarao recently showed that, for $S>0$ and $n=4 S$ +1 , the subgroup actually splits off as a direct summand. In this note we show this to be true for the class of integers $n$ such that $n+1 \neq 2^{r}$. This result is also known to James [2].

In what follows $(\Pi, n)$ will denote an Eilenberg-MacLane space such that $\Pi_{i}((\Pi, n))=0$ for $i \neq n$ and $\Pi_{n}((\Pi, n))=\Pi$. $\Pi$ will always be a cyclic group. $\alpha_{n}$ will denote a generator of $\Pi_{n}((\Pi, n))=\Pi_{n}(\Pi, n)$ and $X$ will be the space $S^{n} \cup_{g} e^{2 n}$ where $g=\left[\iota_{n}, \iota_{n}\right]$.

It is a classically known fact that the kernel of $\Sigma: \Pi_{2 n-1}\left(S^{n}\right)$ $\rightarrow \Pi_{2 n}\left(S^{n+1}\right)$ is the subgroup generated by $\left[\iota_{n}, \iota_{n}\right]$. We thus have the following short exact sequence for odd $n$,

$$
0 \rightarrow Z_{2} \rightarrow \Pi_{2 n-1}\left(S^{n}\right) \rightarrow \Pi_{2 n}\left(S^{n+1}\right) \rightarrow 0 .
$$

In order to split this sequence, all we need to do is find a homomorphism $f: \Pi_{2 n-1}\left(S^{n}\right) \rightarrow Z_{2}$ which is nonzero on $\left[\iota_{n}, \iota_{n}\right]$. Once the sequence is split we have $\Pi_{2 n-1}\left(S^{n}\right)=Z_{2} \oplus \Pi_{n-1}^{S}$ where $\Pi_{n-1}^{S}$ is the $(n-1)$ st stable stem of homotopy groups of spheres.

2. The result. For $n+1 \neq 2^{r}$, there is an Adem relation of the form $\mathrm{Sq}^{n+1}=\sum_{i=1}^{k} a_{i} b_{i}$ where $a_{i}$ and $b_{i}$ are elements in the $(\bmod 2)$ Steenrod Algebra [4, p. 2]. We need the following

Lemma. For $n$ odd, $n+1 \neq 2^{r}, \mathrm{Sq}^{n+1}=\sum_{i=1}^{k} a_{i} b_{i}$ as above, and no $a_{i}=\mathrm{Sq}^{1}$.

Proof. We only have to rule out the term $\mathrm{Sq}^{1} \mathrm{Sq}^{n}$ since any term such as $\mathrm{Sq}^{1}\left(\mathrm{Sq}^{p} \mathrm{Sq}^{r}\right)$ can be regrouped as $\left(\mathrm{Sq}^{p+1}\right) \mathrm{Sq}^{r}$ to serve our ends. For this purpose, notice $\left[4\right.$, p. 11] that $\left\{\mathrm{Sq}^{2}{ }^{i}\right\}$ generates the $(\bmod 2)$ Steenrod Algebra, as an algebra. Thus $\mathrm{Sq}^{n}, n$ odd, can be broken up as

$$
\sum_{a_{i}+b_{i}=n} \mathrm{Sq}^{a_{i}} \mathrm{Sq}^{b_{i}}, \quad \text { where } n>\operatorname{deg} b_{i}>0
$$

Received by the editors August 22, 1967. 
So

$$
\mathrm{Sq}^{1} \mathrm{Sq}^{r}=\sum_{a_{i}+b_{i}=n} \mathrm{Sq}^{a_{i}+1} \mathrm{Sq}^{b_{i}}
$$

and the lemma is proved.

The above Adem relation may thus be regrouped in such a way that no $a_{i}=\mathrm{Sq}^{1}$. We now use this Adem relation to construct a Postnikov tower which is a universal example for the secondary cohomology operation associated to our particular grouping in the Adem relation. The operation is a modulo 2 operation defined on integral cohomology classes. In the diagram below, $\operatorname{deg} b_{i}$ refers to the ordinary degree of $b_{i}$ as an element in the Steenrod Algebra.

$$
\begin{aligned}
& \left(Z_{2}, 2 n-1\right) \stackrel{i_{2}}{\rightarrow} E_{2} \\
& \begin{aligned}
\prod_{i=1}^{k}\left(Z_{2}, n+\operatorname{deg} b_{i}-1\right) \stackrel{i_{1}}{\rightarrow} & E_{1} \underset{P_{2}}{\rightarrow}\left(Z_{2}, 2 n\right) \\
\downarrow & \stackrel{P_{1}}{P_{1}}
\end{aligned} \\
& (Z, n) \underset{\underset{i=1}{\times} b_{i}}{\longrightarrow} \prod_{i=1}^{k}\left(Z_{2}, n+\operatorname{deg} b_{i}\right)
\end{aligned}
$$

Consider the homotopy groups of the total space $E_{2} . \Pi_{n}\left(E_{2}\right) \supset Z$ on a generator $\eta$ such that $p_{1 f}\left(p_{2 f}(\eta)\right)=\alpha_{n} \in \Pi_{n}(Z, n)$ and $\Pi_{2 n-1}\left(E_{2}\right)=Z_{2}$ by some diagram-chasing together with a check on the integers $\operatorname{deg} b_{i}$ using Lemma 1.

The secondary operations, $\Phi$, defined above are particular examples of a class of operations considered in [1] by Brown and Peterson, where the following is proved:

ThEOREM. If $u \in H^{n}(X ; Z)$ is a generator and $\Phi(u) \neq 0$, then $[\eta, \eta] \neq 0$.

Brown and Peterson then show that, for the sort of $\Phi$ constructed above, $\Phi(u) \neq 0$.

Now let $S_{n} \stackrel{f^{\prime}}{\rightarrow}(Z, n)$ represent $\alpha_{n}$. Trivially this map lifts to a map $S_{n}^{n} \stackrel{f}{\rightarrow} E_{2}$ which represents $\eta$. But the naturality of Whitehead products gives us $f_{\#}\left(\left[\iota_{n}, \iota_{n}\right]\right)=\left[f_{\sharp}\left(\iota_{n}\right), f_{\#}\left(\iota_{n}\right)\right]=[\eta, \eta] \neq 0$, so that we have

THEOREM 1. There is a space $E_{2}$ and a map $f: S^{n} \rightarrow E_{2}$ so that the induced map on homotopy groups takes $\left[\iota_{n}, \iota_{n}\right] \in \Pi_{2 n-1}\left(S^{n}\right)$ to a nonzero element in $\Pi_{n}\left(E_{2}\right)=Z_{2}$, when $n+1 \neq 2^{r}$ and $n$ is odd.

This splits the sequence in the introduction and establishes the claim made there. 


\section{REFERENCES}

1. E. H. Brown, Jr. and F. P. Peterson, Whitehead products and cohomology operations, Quart. J. Math. Oxford Ser. (2) 15 (1964), 116-120.

2. I. M. James, Review of [3], Math Reviews 34 (1967), \#811.

3. G. V. Krishnarao, Note on a Whitehead product, Proc. Amer. Math. Soc. 17 (1966), 1131-1132.

4. N. E. Steenrod and D. B. A. Epstein, Cohomology operations, Princeton Univ. Press, Princeton, N. J., 1962.

NORTHEASTERN UNIVERSITY 